\title{
The Method for Removing Rain in Multiple Images
}

\author{
Hong $\mathrm{Ji}^{\mathrm{a}}$ and Zhen Chen ${ }^{\mathrm{b}, *}$ \\ College of Computer Science and Technology in Beihua University, Jilin 132013 \\ a349522267@qq.com, ${ }^{b} 59975235 @ q q . c o m$
}

\begin{abstract}
Keywords: Rain removal; Image recovery; Wavelet multi-level decomposition; Wavelet fusion
\end{abstract}
\begin{abstract}
The outdoor vision system under the condition of rain weather, especially when the intensity of rain is relatively high, the contrast of the images obtained is low and unclear which resulting in serious degradation. The traditional way to remove the rain is restricted by its strength, and the result is not satisfactory. According to the feature about that the vision system can obtain a plurality of consecutive different degraded images in a short time, this paper deals with multiple images to achieve the recovery. And according to the dynamic characteristics of rain and snow, the direction, strength, and shape of rain lines or snow lines are not fixed. It is difficult to establish a unified physical model in the spatial domain, but they are not affected by their dynamic characteristics in the frequency domain. This paper analyzes from the perspective of frequency domain, uses wavelet multi-level decomposition and wavelet fusion method to determine the specific layer of rain noise and formulates a fusion rule based on the degree of rain noise pollution. A certain number of layers of continuous degraded images are wavelet-fused to achieve the purpose of removing rain (snow). The simulation results show that the method proposed in this paper has satisfactory recovery results and is not subject to noise intensity.
\end{abstract}

\section{Introduction}

With the rapid development of computer technology, the application of outdoor vision systems has become more and more widespread. It plays an important role in the areas of target identification and tracking, traffic monitoring, remote sensing monitoring, and Military reconnaissance. However, under the adverse weather conditions, the robustness and practicality of outdoor vision systems have been greatly affected. Especially in rain weather, the acquired image has a high degree of pollution, and the overall image looks vague, and the detailed content is unclear, and it cannot be applied to features extraction and target recognition. Therefore, it is of great significance to clear the image acquired under adverse weather, especially rain weather conditions, so as to make the outdoor visual system more reliable and adaptable.

There are two major methods for recovering rain images: One is a hardware-based method: In 2005, Garg [1,2] et al. used video camera parameters to reduce the amount of rain in the video. This method is difficult to apply to the outdoor vision system. Moreover, in the case of heavy rain (snow), the clearness effect is not ideal, so there is less research on the hardware method. The other one is a software-based approach: one or more degraded images are processed through appropriate digital image processing algorithms to remove the rain (snow), including methods based on the image spatial domain and based on image frequencies domain. The former started earlier and the research was also deeper: In 1999 Hase [3] et al. proposed a method that can reduce the visibility of snow in video in real time: median filtering was performed on each pixel in the time axis direction. In 2004, Garg[2,4] and others studied the optical and kinematic characteristics of raindrops to construct an optical model and a kinetic model of raindrops to detect and remove raindrops, which is called the frame difference method. In 2006, Zhang et al. [5] used k-means clustering on all pixels with the same coordinates in the time axis of the video to detect and remove raindrops, which is called a clustering method. The after method of rain based on the image spatial domain is basically an improvement of the frame difference method or the clustering method. In 2008, Brewer [6] et al. detected raindrops through five consecutive frames of images and constructed a new method for replacing raindrop pixels. In 2008, Zhao Xudong [7] and others studied the range of brightness distribution of raindrops and used it to determine whether a pixel was covered by 
raindrops. The method based on image frequency domain started late. In 2009, Barnum [8, 9] and others constructed the frequency domain model of raindrops and identified raindrops through this model, and then performed filtering operations to remove the rain. However, the process of constructing the frequency domain model of raindrops is quite complicated. In 2011, Fu [10] et al. performed sparse coding decomposition on a single degraded image, and also remove the rain through a filtering operation. As a result of single image sharpening, the loss of image details is inevitable.

This paper deals with multiple images to achieve the recovery. And according to the dynamic characteristics of rain and snow, the direction, strength, and shape of rain lines or snow lines are not fixed. It is difficult to establish a unified physical model in the spatial domain, but they are not affected by their dynamic characteristics in the frequency domain. This paper analyzes from the perspective of frequency domain, uses wavelet multi-level decomposition and wavelet fusion method to determine the specific layer of rain noise and formulates a fusion rule based on the degree of rain noise pollution. A certain number of layers of continuous degraded images are wavelet-fused to achieve the purpose of removing rain (snow). The simulation results show that the method proposed in this paper has satisfactory recovery results and is not subject to noise intensity.

Since rain is a dynamic adverse weather, it is possible to obtain a plurality of successively different degraded images through the outdoor vision system in a short time.

\section{Digital Image and Wavelet Analysis}

The digital image is essentially a two-dimensional discrete signal whose resolution is limited. In the early stage, the image can be converted between the spatial domain and the frequency domain through the Fourier transform, and unnecessary frequencies in the image can be removed. However, with the deepening of the research on digital images, the limitations of Fourier transform in solving certain problems are gradually exposed: It is a kind of global transformation, and the overall spectrum of the image is obtained, so the local characteristics of the spatial domain of the image cannot be expressed. It cannot accurately find the specific frequency of rain in the image. However wavelet analysis is like a microscope. It is a quantitative image analysis method that can determine the frequency range of rain in the image through wavelet multi-layer decomposition.

Wavelet analysis can perform row and column filtering and high and low pass filtering respectively. The first layer of low frequency myopia coefficient $\mathrm{C}_{1}$ was obtained by row and column low pass filtering on the original image, which mainly includes background and color information in the image. In the same way, row, column, row and column high-pass filtering are performed on the original image, and obtained the first level of high-frequency detail coefficients $D_{1}^{\mathrm{H}}$, vertical high-frequency detail coefficients $\mathrm{D}_{1}^{\mathrm{V}}$, and diagonal high-frequency detail coefficients $\mathrm{D}_{1}^{\mathrm{D}}$, which mainly contain texture and edge information in different directions of the image. Then repeat the above operation on $\mathrm{C} 1$ to get $\mathrm{C} 2, \mathrm{D}_{2}^{\mathrm{H}} 、 \mathrm{D}_{2}^{\mathrm{V}} 、 \mathrm{D}_{2}^{\mathrm{D}}$ which are the low-frequency coefficients of the second layer and the high-frequency coefficients of the three directions, respectively. If you continue to filter the low-frequency coefficients of the m-1 layer, you can get $\mathrm{Cm}, \mathrm{D}_{\mathrm{m}}^{\mathrm{H}} 、 \mathrm{D}_{\mathrm{m}}^{\mathrm{v}} 、 \mathrm{D}_{\mathrm{m}}^{\mathrm{D}}$ in the same way. The relation of its frequency is: $\mathrm{Cm}<\mathrm{Cm}-1<\mathrm{Dm}<\mathrm{Dm}-1$.

The rain noise and degraded image scene information are different in frequency. Among them, rain noise occupies a relatively high frequency, texture details and edge details in scene information also occupy higher frequencies, sometimes even higher than rain noise, The background and color information of the image and scene is the lowest frequency. So in an image that contains rain noise, the frequencies are made up roughly like these: The frequency of background and color information of image scene is the lowest, the frequency of rain noise is high, and the texture and edge of image scene details are the highest. By using wavelet analysis to multi-layer decompose a degraded image, rain noise should be included in the lower-layer high-frequency coefficients, in order to ensure that the decomposed image details contain as little rain noise as possible. Generally take a larger number of decomposition layers. Then determine the specific layers of rain noise, image details, image 
background information and color information.

In the following, a rainy day image (see Figure 1a) is decomposed by wavelet multi-layer decomposition. The number of decomposition layers is chosen as 10. Fig. $1 \mathrm{~b}$ shows the reconstructed image of the low-frequency coefficient of the 10th layer, Fig. 1c to Fig. 11 show the reconstructed image of the high-frequency coefficient from the 10th layer to the 1st layer, and Fig. $1 \mathrm{~m}$ shows the 10th layer to the 5th layer and the high frequency coefficients of 1st layer, which contains very little rain noise. Figure $1 \mathrm{n}$ is the reconstructed image of the high-frequency coefficient from $4^{\text {th }}$ layer to layer 2 nd layer. It contains most of the rain noise.
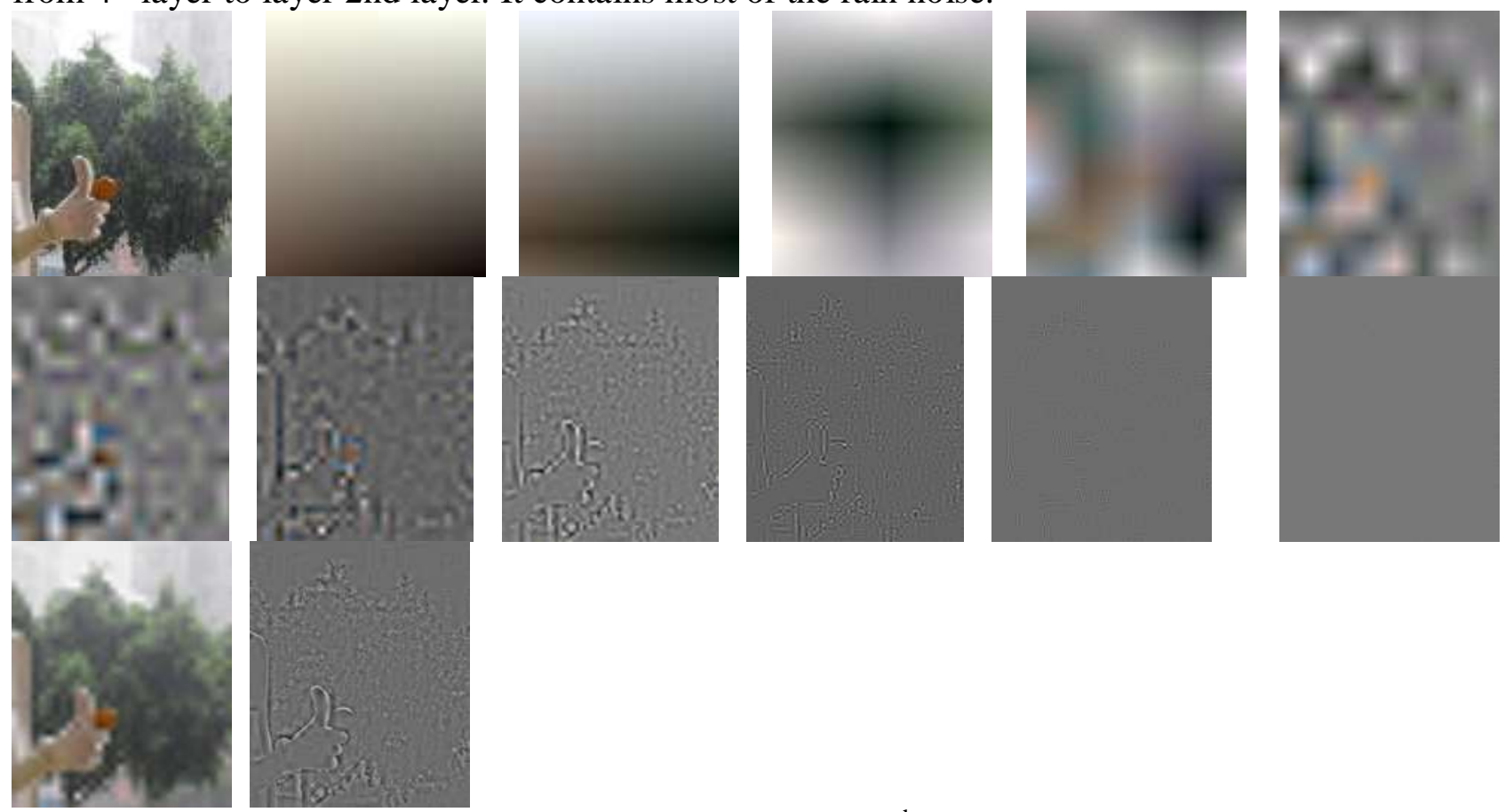

(a)High frequency reconstruction of rain images (b) $10^{\text {th }}$ layer low-frequency reconstruction (c) $10^{\text {th }}$ layer high-frequency reconstruction (d) 9th layer high-frequency reconstruction (e) 8th layer high-frequency reconstruction (f) 7th layer high-frequency reconstruction ( $g$ ) 6th layer high-frequency reconstruction ( $\mathrm{h}$ ) 5th layer high-frequency reconstruction ( $i$ ) 4th layer high-frequency reconstruction ( $\mathrm{j}$ ) 3rd layer high-frequency reconstruction ( $\mathrm{k}$ ) 2nd layer high-frequency reconstruction (1) 1 st layer high-frequency reconstruction $(\mathrm{m})$ No rain image ( $\mathrm{n}$ ) Rainy image

Fig. 1 (a-n) Wavelet multi-level decomposition of rainy images

\section{Fusion of Multiple Consecutive Degraded Images}

Rain days are dynamic adverse weather. Under such conditions, outdoor vision systems can easily collect multiple consecutive degraded images. Next, these consecutive degraded images must be wavelet-fused to remove rain noise. According to the frequency structure of the degraded rain image analyzed above, the background and color information, rain noise, texture details, and edge detail information should be fused separately, and the appropriate fusion rules should be formulated according to the three different characteristics. .

The fusion of rain noise, that is, the fusion of high-frequency coefficients from the 2nd layer to the 4th layer, for these coefficients, Whether the fusion rules are reasonable or not plays a vital role in effectively removing rain noise from degraded images. Then analyze the characteristics of raindrops. Because raindrops move fast, they appear as high-brightness thin lines in video images. The raindrop image we see is the result of reflection and internal refraction of light at its surface, which is why the brightness of the raindrop is high. As shown in Figure 2, the ambient light is collected by the vision system after being reflected by the raindrops' spherical and internal refraction of the raindrops. Whichever refraction or reflection, the maximum angle of light that can 
be collected by the system is 165 degrees [4]. In this way, enough light is collected through the raindrops, and the brightness of the raindrops has been greatly improved. So although the raindrop is transparent, its brightness does not depend on the background brightness it covered. Through further experiments, it is proved that the intensity of the raindrops is not only independent of the background it covered, but the intensity of the raindrops is basically the same. As shown in Figure 3, the left side is the raindrop and background, and the background is composed of 5 horizontal stripes with different brightness.

The right side is a function image of pixel brightness and time. The brightness increases when the background is covered by raindrops, and the brightness values are basically the same (around 200). Therefore, high brightness and consistent brightness are two important characteristics of raindrops.

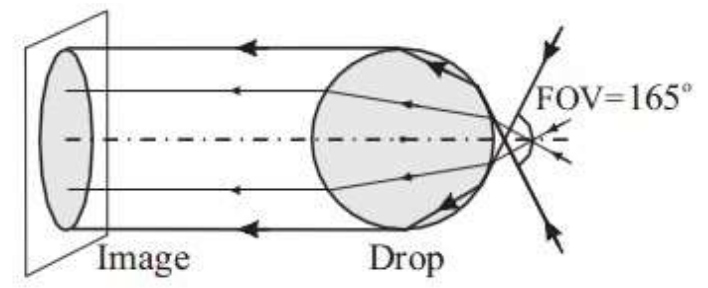

Figure 2 Light reflected by the surface of the raindrop and internal refraction
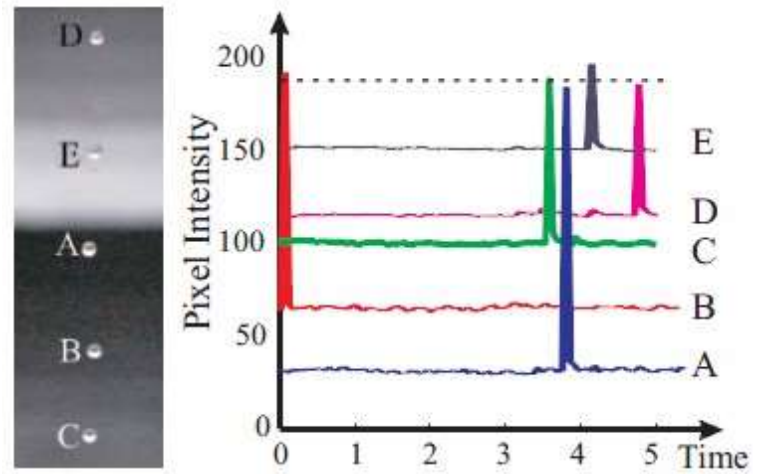

Figure 3 experiment about the relationship between raindrop brightness and background

Next, analyze the characteristics of the snowflake. Although snowflakes are opaque and move slower than raindrops, but snowflake also has similar characteristics with raindrops. First, the brightness of snowflakes is high. Second, the brightness of all snowflakes is basically the same. According to the characteristics of rain noise, wavelet fusion is performed on the layers containing rain noise, and formulated the specific fusion rules. Since the brightness of the rain is significantly higher than the brightness of the surrounding background, thus, the gray scale of the pixels covered by rain and background pixels varies greatly which can produce better edge effect. we can use local gradients to measure the degree of change in gray scale, defined as following: $G=\frac{1}{M \times N} \sum_{i=1}^{M} \sum_{j=1}^{N} \sqrt{\Delta x f(i, j)^{2}+\Delta y f(i, j)^{2}}$

Where $\mathrm{G}$ is the local gradient, $\Delta x f(i, j)$ and $\Delta y f(i, j)$ are the gradient values of the points $(\mathrm{i}, \mathrm{j})$ in the horizontal and vertical directions respectively, $\mathrm{M}$ and $\mathrm{N}$ are the length of the local area sides. Since the brightness of rain does not change, the pixels covered by rain have higher and more stable energy than the background pixels. The local energy can be used to measure the size of the pixel energy and is defined as follows:

$$
E=\frac{1}{M \times N} \sum_{i=1}^{M} \sum_{j=1}^{N} f(i, j)^{2}
$$

Where $\mathrm{E}$ is the local energy, $\mathrm{f}(\mathrm{i}, \mathrm{j})$ is the gray value of the pixel, and $\mathrm{M}$ and $\mathrm{N}$ are the lengths of the local area sides. Local gradients have directionality and local energy has stability. These two parameters can reflect the high and consistent brightness of rain noise. Multiply $\mathrm{G}$ and $\mathrm{E}$ to get a new parameter $S$ which called the degree of rain noise pollution, which can further amplify the 
characteristics of rain noise so as to remove rain noise.

\section{Wavelet Fusion Method Based on the Degree of Rain Noise Pollution}

The above definition of $\mathrm{S}$ (i.e., rain noise pollution) in the image is well said the degree of rain pollution, which can effectively distinguish between rain areas and background areas in the image. Therefore, this paper proposes a wavelet fusion method based on the degree of rain noise pollution. The specific steps are as follows:

(1) Wavelet decomposition. the 10-layer wavelet decomposition is performed on the degraded image to obtain high-frequency coefficients from the 4th layer to the 2 nd layer, and rain noise is mainly contained in it

(2) Fusion of the high frequency coefficients which including rain noise. The coefficient matrix corresponding to the high-frequency coefficients in each direction of the 4th layer to the 2nd layer is obtained, and then, calculated the $\mathrm{S}$ matrix of pollution degree which corresponding to these coefficient matrices (local area is $3^{*} 3$ ), and these $\mathrm{S}$ matrices is normalized to obtain the new $\mathrm{S}$ 'matrix. In these $S$ ' matrices, the larger the numerical value at the same position, the more serious polluted by rain noise in these positions. Therefore, each coefficient matrix is inversely weighted with each S' matrix to obtain the fused coefficient matrix. The rain noise will be greatly reduced. In order to further reduce rain and snow noise, a weight less than 1 can be set for the fused coefficient matrix (0.9 in this paper).

(3) Fusion of coefficients that do not include rain noise. It is the fusion of the coefficients of the 10th to 5th layers and the high frequency coefficients of the 1st layer. These coefficients contains the main effective information in the image, Therefore, it can be processed by means and set a greater weight than 1 for the fused coefficient matrix (1.2 in this paper), further highlighting the color field and details of the image.

(4) Wavelet reconstruction. The coefficients of each fused part were reconstructed to obtain the fused image without rain .

The single fused image obtained through the above steps can well remove the rain noise in multiple consecutive images, but it cannot remove the rain fog in the non-rain region. However, when the rain is often accompanied by the appearance of fog, the details of the image are blurred and the contrast is reduced, so we must be process the fog. In this paper, based on the dark channel priority [11], proposed an improved single-image defogging algorithm to quickly defog a single image with fog after rain (snow). Using a fast bilateral filtering method, a detailed and clear dark channel is calculated, and a transmission image is estimated based on the physical model of fog imaging. Compared with the traditional algorithm, the estimated transmission image is meticulous and clear with no optimization required, thus overcoming the shortcomings of the traditional algorithm to optimize the transmission image with a lot of time, greatly reducing the complexity of the algorithm, and achieved fast and high quality defogging of single image. The fused image with the process of defogging has been greatly improved in detail definition and color fidelity.

\section{Simulation Analysis}

In order to verify the practicability and validity of the algorithm, we use Matlab 2012a to perform simulation experiments on an ordinary PC with the operating system of Windows XP and a CPU with AMD quad-core $3.0 \mathrm{GHz}$ and 4GB RAM. In this paper, three scenarios of heavy rain scene, moderate rain scene and heavy snow scene are selected. The frame difference method, clustering method and the method proposed by this paper are used for processing. This method fuses 9 frames of continuous images and replaces the 5th frame with the fused clear images to realize removing of the rain in the entire video. The experimental results are shown in Figure 4. 


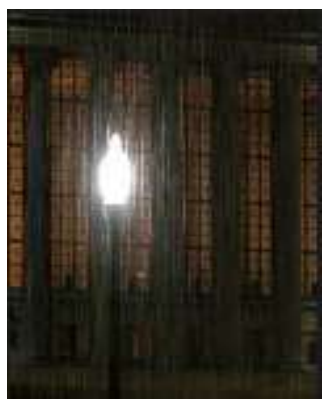

(a) Heavy rain image

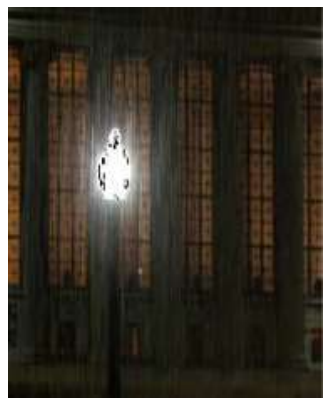

(c) Clustering result

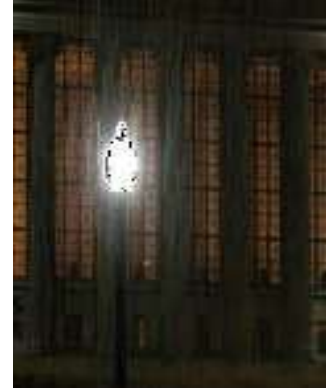

(b) Frame difference method result

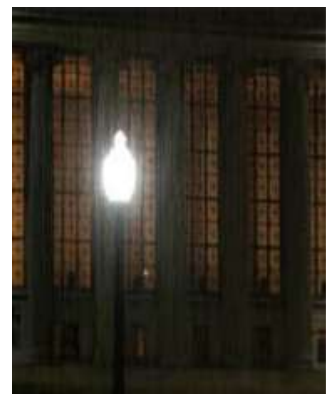

(d) Result of this paper

Figure 4 (a-d) Various Scene Processing Results and Comparison

Figure 4 (a) is the original heavy rain, moderate rain, heavy snow image; Figure 4 (b) are the results of the frame difference method; Figure 4 (c) are the results of the clustering method; Figure 4 (d) are the processing results of the method proposed in this paper. From the experimental results: Although the frame difference method and the clustering method are satisfactory to the middle rain, but the results of heavy rain, especially heavy snow, are not satisfactory. However, for the degraded image of rain with high intensity, this method proposed in this paper has also achieved very satisfactory recovery results because the direction, strength and shape of the rain line are not fixed and It is difficult to establish a unified physical model in the spatial domain, but the analysis of rain in the frequency domain is not affected by their dynamic characteristics. This paper adopts wavelet multi-level decomposition and wavelet fusion method to process multiple continuous degraded images from the frequency domain perspective.

\section{Conclusion}

According to the dynamic characteristics of rain (snow), the trajectory direction, intensity, and shape are not fixed. It is difficult to establish a unified physical model in the spatial domain. However, in the frequency domain, they are not affected by their dynamic characteristics. Adopting wavelet multi-level decomposition and wavelet fusion methods: first determine the specific layer of rain noise, and then formulate a fusion rule based on the degree of rain noise pollution, and using the wavelet fusion for a specific layer of multiple successively degraded images to reach the purpose of removing rain (snow). From the simulation results, this method is not constrained by rain strength, and the recovery result is satisfactory, which is better than the traditional frame difference method and clustering method.

\section{Acknowledge}

This paper is supported by the 13th Five-Year planning project for the education department in Jilin province to technology research (A new algorithm of rain removal in video, Grant JJKH20180343KJ) and the project for training outstanding youth of Jilin (Grant 20156412), of which Chen Zhen is the project leader. So Chen Zhen is the corresponding author of this paper. 


\section{References}

[1] K. Garg and S. K. Nayar, "When does a camera see rain", Proc.ICCV, Oct. 2005, vol. 2, pp. 1067-1074.

[2] K GARG,S K NAYAR, "Vision and rain[J] ",International Journal of Computer Vision,2007,75( 1) : 3-27.

[3] H HASE,K MIYAKE,M YONEDA, "Real time snowfall noise elimination", IEEE Proceedings of 1999 International Conference on Image Processing. 1999,406-409.

[4] K. Garg and S. K. Nayar, "Detection and removal of rain from videos" ,Proc. CVPR, June 2004, vol. 1, pp. 528-535.

[5] X. Zhang, et al., "Rain removal in video by combining temporal and chromatic properties" , Proc. ICME, July 2006, pp. 461-464.

[6] NBREWER,LIU NIAN JUN, "Using the shape characteristics of rain to identify and remove rain from video", Lecture Notes in Computer Science. 2008,5342: 451-458.

[7] ZHAO XU DONG,LIU PENG,LIU JIAN FENG,ETAL, "The application of histogram on rain detection in video",proceedings of the 11th Joint Conference on Information Science. 2008,345-353

[8] P BARNUM,T KANADE,S NAR, "Spatio-temporal frequency analysis for removing rain and snow from videos", Workshop on Photometric Analysis For Computer Vision. 2007, 14-21.

[9] P. C. Barnum, S. Narasimhan, and T. Kanade, "Analysis of rain and snow in frequency space", IJCV, vol. 86, no. 2-3, pp. 256-274, 2010.

[10] Yu-Hsiang Fu, Li-Wei Kang, Chia-Wen Lin,ETAL, "Single-frame-based rain removal via image decomposition", IEEE International Conference on Acoustics, Speech, and Signal Processing. 2011,178-186

[11] He Kaiming,Sun Jian,Tang Xiaoou, "Single image haze removal using dark channel prior",IEEE Conference on Computer Vision and Pattern Recognition, 2009:1956-1963

[12] Sun Yankui, "Wavelet Analysis and Application ”, Beijing: Machinery Industry Press, 2005. 